\title{
(Un)Frayling design research in design education for the 21Cth
}

\author{
Fernando Galdona, Ashley Hall ${ }^{b}$ \\ a Royal College of Art, London, UK \\ b Royal College of Art, London, UK \\ *Corresponding author e-mail: Fernando.galdon@rca.ac.uk
}

\begin{abstract}
:
This paper will focus on redefining design research education for the 21 Cth by reviewing seminal theoretical work in the field of design by Archer (1968), Cross (1983), Jones (1970), and contemporary critiques such as Herriott (2019), or Galdon \& Hall (2019). In this context, we will contextualise critical issues emerging from Christopher Frayling's seminal paper Research in art and design. By implementing an historical account into previous work, we will understand why Frayling did what he did, why he articulated his framework in the way he did, and the fundamental problems arising from it. In the process, building from previous work of the authors, this paper repositions the ontological nature of design knowledge around notions of prospectivity, abductivity, and probabilism. This position emancipates design from the present, thus overcoming the scientific/tacit paradigms, and liberates design to operate in its future-led prospective and transformational nature. This position aligns with Blauvert's (2008), third wave of design focused on a multiplicity of contingent, boundaried and conditional solutions.
\end{abstract}

Keywords: abductive reasoning, prospective, probabilistic knowledge, theory

\section{Introduction}

As stated in a recent call for papers from the Art History network;

The Debates surrounding and inquiring into the nature of Artistic and Design Research have been in progress since the early $90^{\prime} \mathrm{s} . .$. and still constitute a live interesting area of interest, however, research on design and art continues to constitute a contested academic field. (Guerra, 2021).

This statement positions the origin of artistic and design research with Christopher Frayling seminal paper Research in art and design presented in 1993, however, previous research by Archer (1968), or Cross (1983) seems to be absent in this account. This paper will focus on design research evolution to review what happened before Frayling's seminal paper to contextualise why Frayling did what he did, why he articulated his framework in the way he did, and the problems emerging from his account. 
From this point, we will review contemporary critiques (Harriot, 2019; Galdon, 2019a) to underpin the problems that Frayling' model create for today's research in Design. This process will support a different ontological nature for design knowledge (Galdon, 2019a), which aims to clarify practicebased research projects in which experimentation, reflection, critical practices and analysis are taking place simultaneously. This clarification aims to facilitate cross-culture collaboration by proposing a distinctive model that can be understood by designers, humanists, and scientists.

Transformational processes embodied around the notion of World-making involve this generative interweaving between practices and forms, methodologies and phenomena, doings and knowledges. However, the missing ontological acknowledgement positioned research in design as a contested academic field creating confusion among practitioners. The institutional paradigm of linear, arborescent, cataloguing research is now challenged by a clear model where practice shape fragmented territories, in dialogue, in solidarity, collectively constituted, and transformational.

\section{Discussion}

\subsection{Bruce Archer}

Historically, design approaches in research have been compared to and categorised within the sciences, arts and humanities. For instance, C.P. Snow (1959) defined the separation of the domains of knowledge into the sciences and the arts and humanities. However, the design discipline can be seen as having its own distinct way of understanding the world. Its fundamental approach, based on planning, solution-based problem solving, problem shaping, synthesis and preparedness, readiness and appropriateness in the built environment, determines a different manner of knowing. Therefore, prospective disciplines such as design can be positioned as their own specific practices, distinct from the aforementioned sciences, and humanities. In this context, Bruce Archer (1978) went some way towards proposing design as the third culture of thinking, fulfilling Snow's challenge to fill the vacant plot (Snow, 1959). Archer's doctoral work attempted to explain design as a special branch of science (but, usefully, it failed to do so) (Boyd Davis, 2016).

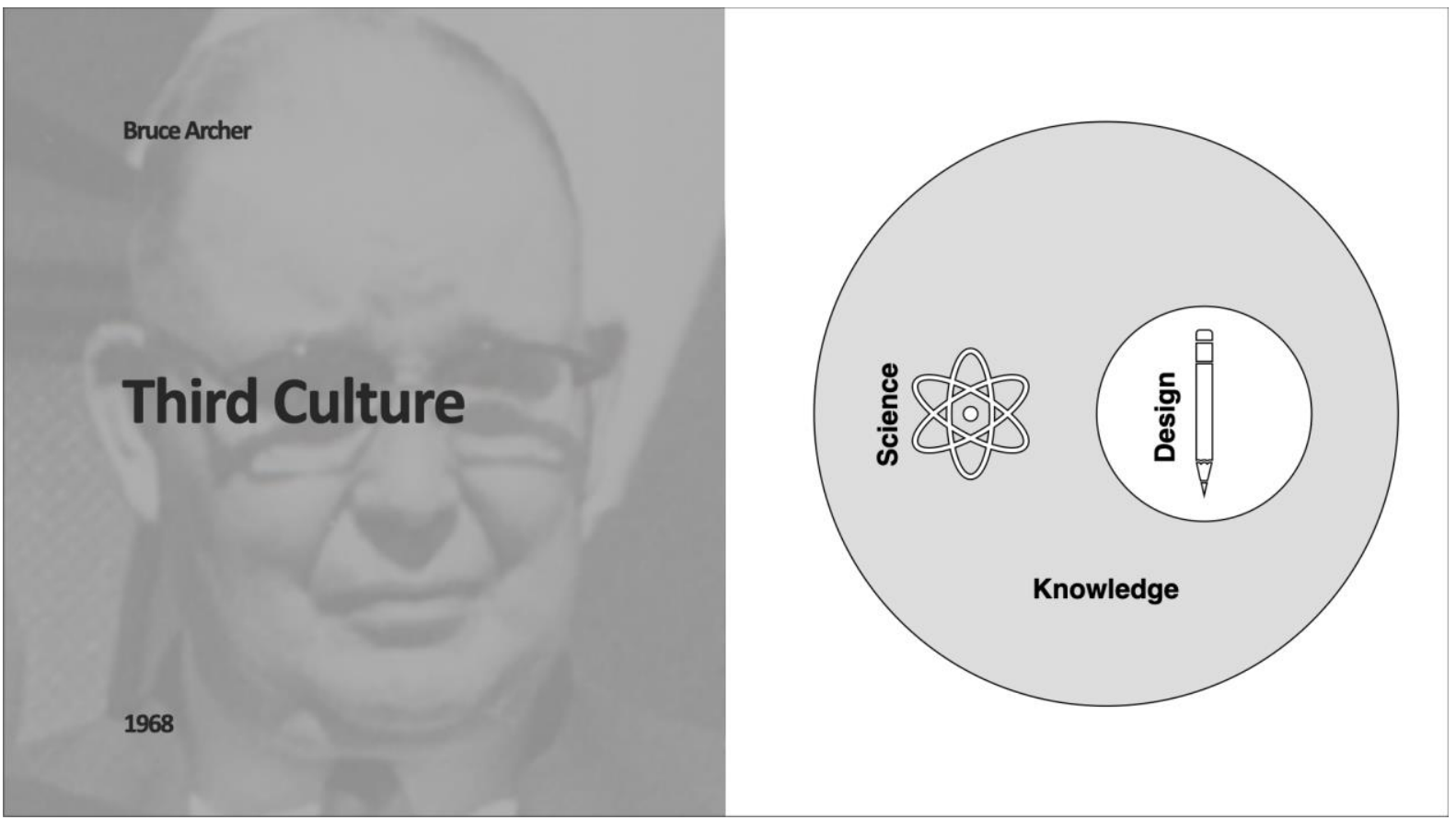

Figure 1. Bruce Archer's doctorate attempted to explain design as a special branch of science. 
Archer's model enters in a contradiction; If design is different from the sciences, then the output cannot be scientific. We characterise this paradox as; the factuality problem.

\subsection{Nigel Cross}

Some years later, Nigel Cross, in his seminal paper Designerly ways of knowing, builds on Archer's work at the Royal College of Art, and describes this third culture as "[...] the collected experience of the material culture, and the collected body of experience, skill and understanding embodied in the arts of planning, inventing, making, and doing"'’. (Cross, 1982, p. 221)

In the process, Cross differentiated design from the sciences and humanities by comparing the terms of the kind of phenomenon that is studied in the three cultures; the sciences focus on the natural world, the humanities on human experience, and design on the human-made world. He also differentiated between the appropriate methods with which to approach each "culture". The sciences use controlled experiments, classification and analysis, while the humanities use analogies, metaphors, criticism, and evaluation. Finally, design uses modelling, pattern-formation and synthesis. In terms of the values of each culture, the sciences aim for objectivity, rationality, neutrality, and concern for "truth", whereas the humanities aim is for subjectivity, imagination, commitment, and concern for "justice". Finally, in design, practitioners aim for practicality, ingenuity, empathy, and concern for "appropriateness"(Cross, 1982, pp. 221-222). (Fig, 2)

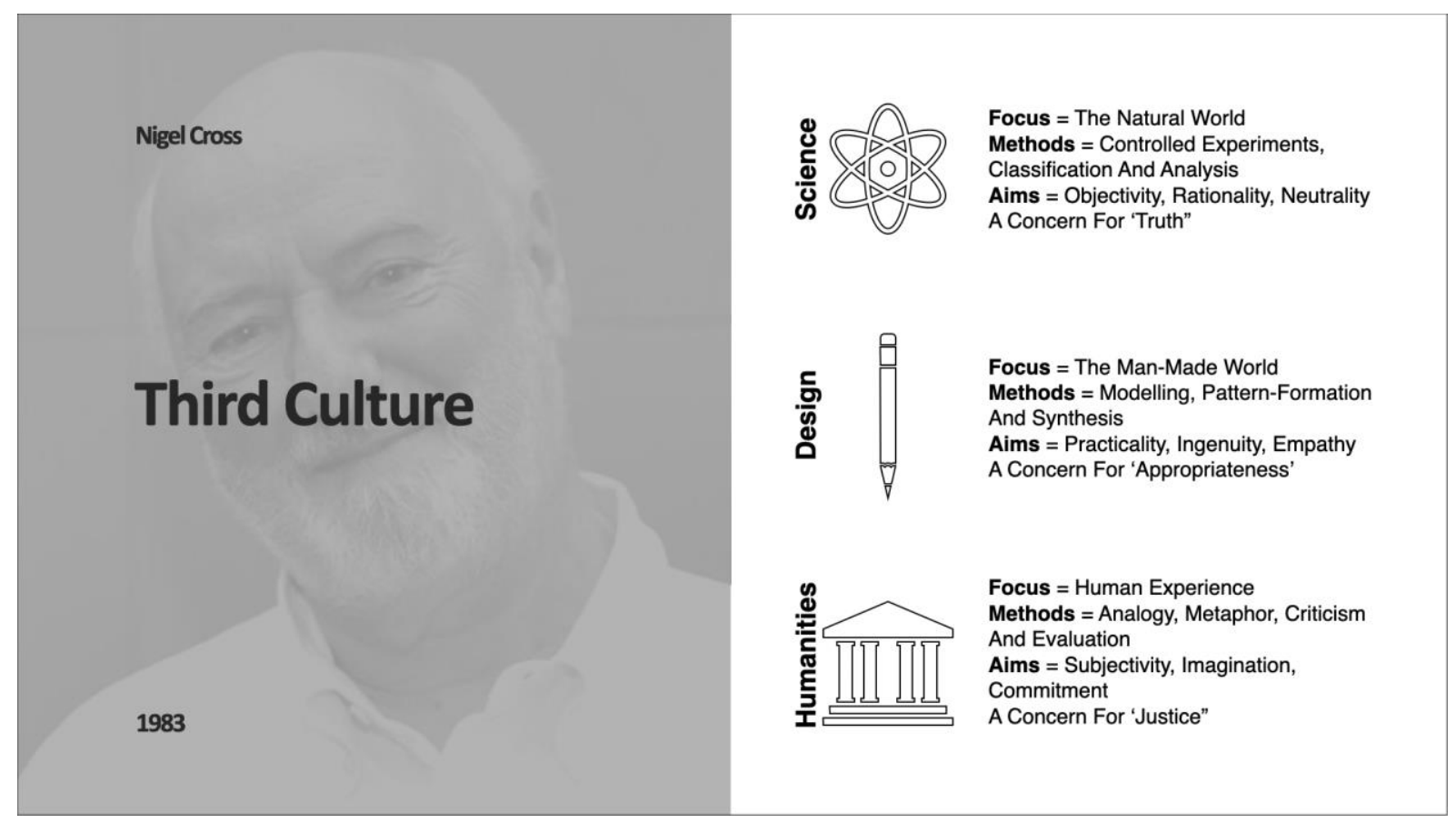

Figure 2. Nigel Cross differentiated design from the sciences and humanities by comparing the terms of the kind of phenomenon that is studied in the three culture.

Cross addressed the issue of factuality (scientific empiricism) by introducing tacit knowledge as an alternative. For Polanyi $(1958,1966)$, who coined the term, this type of knowledge cannot be adequately articulated by verbal or written means. The notion of tacit knowledge aligns perfectly with the practice of design but present a fundamental paradox in the context of research, as research practise and function revolve around transferability. This typology of knowledge created a problem which we characterise as; the transferability problem. 


\subsection{Christopher Frayling}

In this context Christopher Frayling introduced his seminal paper Research in art and design (1993). Consciously or unconsciously, as he did not reference him, He built from Archer by stating that design is different from science (art and design is by no means identical with science (p.3)), and aligned with Nigel Cross in establishing its practices as fundamentally internal by building from Picasso's uncomfortable verbalisation of his work (tacit knowledge rather than propositional (p.3)). On the other hand, Frayling acknowledges later in the paper, that the tacit element may not be sufficient, as it would mean that every artist would be entitle of a Ph.D. As a result, the final output may be a combination of internal and external knowledge (the Cognitive art tradition (p.5)). In order to explain this conundrum, he proposed three modes of research in design (Fig, 3);

- design into art and design

This model refers to traditional models of research. Historical research, Aesthetic research, and Theoretical research. The expected output is a thesis, or dissertation. This approach is focused and more related to history. This model is archive based and is external.

- design through art and design

Frayling points towards materials research, development work research, and action research (diaries instructing step-by-step processes). The main outputs are studio work and a report to communicate the results. This approach is focused on the process.

- design for art and design

In this model thinking is embodied in the artefact, therefore, work is not verbally communicable. The main output is an Artefact. This model represents the embodiment of tacit knowledge. He refers to Picasso's work and points that this model would entail to grant Ph.Ds to the entire history of art. He defines design for as thorny. This approach is internal.

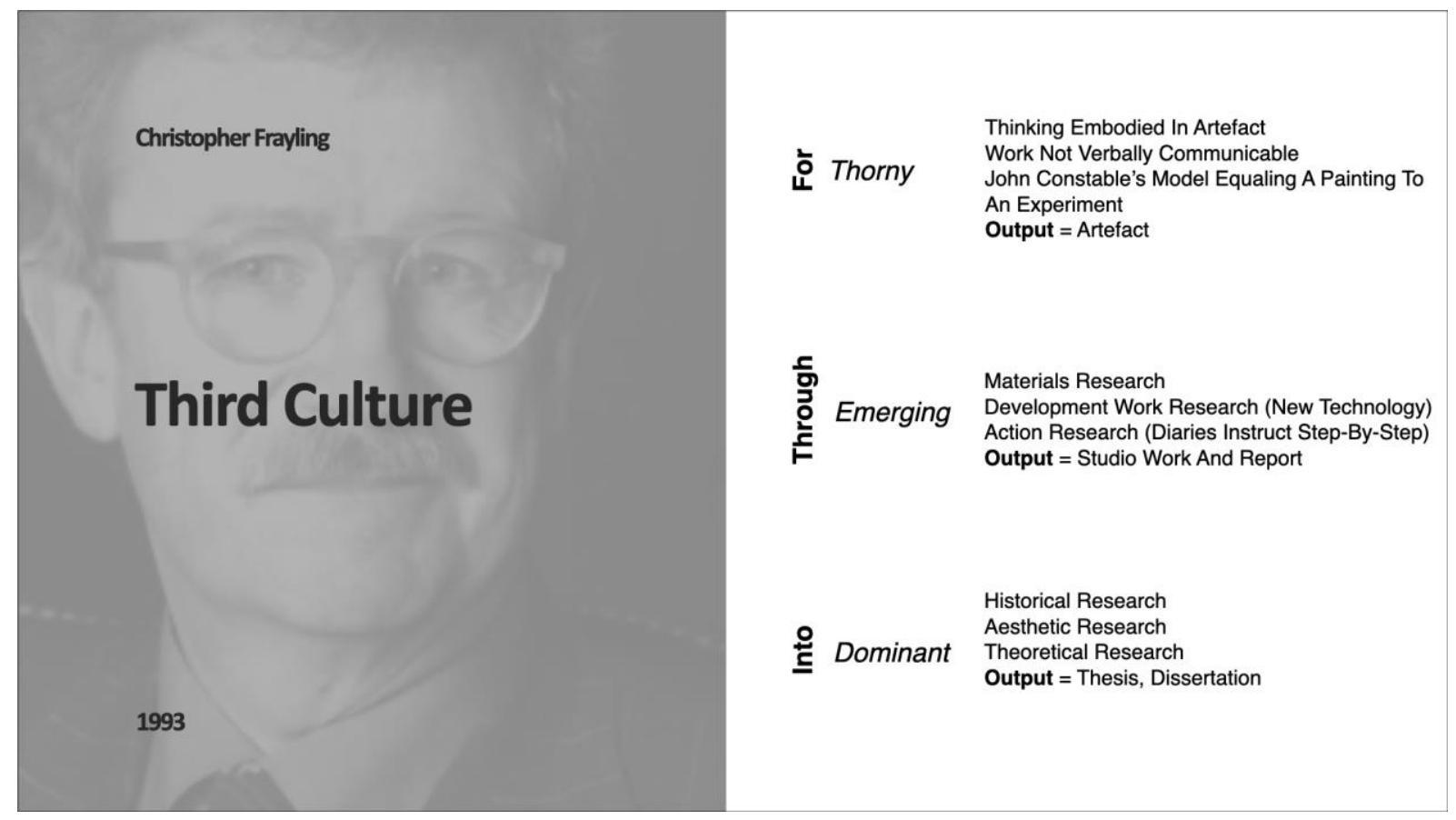

Figure 3. Christopher Frayling three modes of research in design 
From the three perspectives he presents, into is more related to history, and for embodies practise but falls into the untransferable. Only research through seems to be operational in the context of design. In this context its implementation is more related to process and documentation.

The notion of diaries instructing a step-by-step process of a practical experiment in the studio seem contradictory to earlier differentiation of design to scientific enquiry (p.3). In fact, this method seems to suggest repeatability of some kind in the reported process.

The notion of documentation is very interesting, insofar as is supporting novel knowledge. The problem is if you substitute knowledge by collection. As he acknowledges, the act of collecting does not imply per se any contribution to knowledge except if it is novel in itself. But then you are making a contribution to archival research, not to design.

The John Constable's model reported by Frayling which equals a painting to an experiment is very interesting, but as pointed by Picasso, you need to find something. This approach is relevant in design research insofar as the experiment leads to a contribution to knowledge, otherwise is practise. This aspect of a novel contribution to knowledge is missing in the whole paper. This, and the transferability of it, are the fundamental nature of academic research.

Into seems to be more related to history (closer to the humanities than Frayling may suggest). For seems to be the preferred model by practitioners, and through seems to be the most adopted model. For is not operational in the context of research due to the transferability problem (as Frayling acknowledges (thorny)), and the latest arguments reduce research through design to a process (closer to science than Frayling may suggest). I characterise this latest problem as; the procedural problem. These elements question his models to be a constitutive and differentiated field or culture, as suggested by Archer, Cross, or Frayling himself.

\subsection{Richard Herriott}

In what kind of design is research through design? Herriott (2019) question precisely the distinction between "standard" research design and Research through Design. He asks whether research through design is actually different from existing methods of scientific research, and if this assumption is justified.

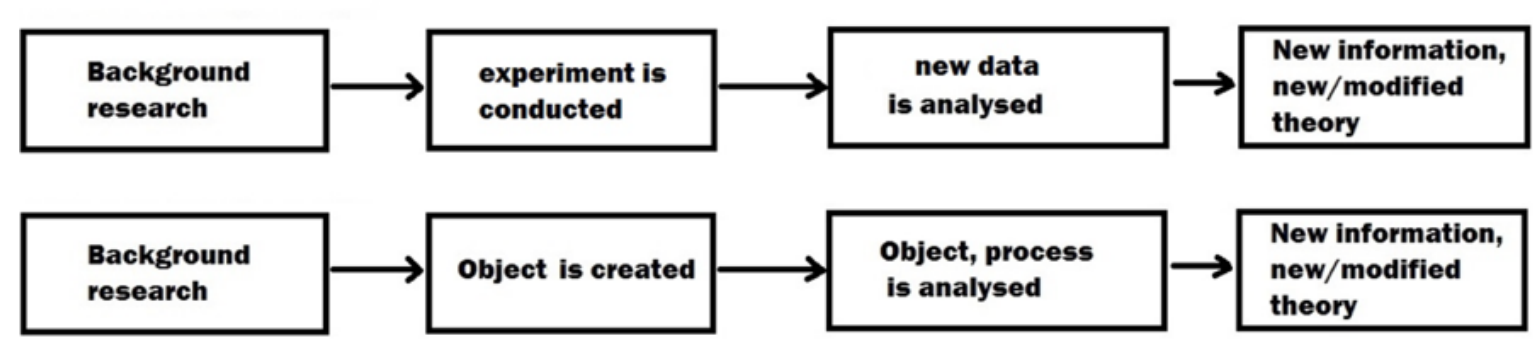

Figure 4. Simplified research into design process versus Simplified research through design process. Herriot, 2019.

In conducting a comparative study (Fig. 4) Herriott underpins how research through design and research in design make objects/processes their object of study. In his account, the main difference is that "in the case of research through design the object is made for the purpose" (Herriott, 2019, p.8). However, the methods of observation and analysis are the same. For Herriott, the main similarity between the modes of research being studied is that both research in design and research through design conduct experiments. In his account the experiment may vary in position but both are artificial situations. 
This analogy extends to design accounts into the natural sciences or social sciences (or a hybrid of both). In both cases, he suggests "a reading of the background theory leads to a research question which is testable by experiment. The difference lies in the extent of and effect of the designer/researcher's role in the design work" (Herriott, 2019, p.8).

In his conclusion, he states that "Research through design is part of a well-established tradition in science of using an experiment of some type to test a hypothesis about X. This procedure is, after all, exactly what practicing designers do too: make some observations about the world, make a hypothesis that the user needs object $\mathrm{X}$ and then test the hypothesis by making a prototype of $\mathrm{X}^{\prime \prime}$ (Herriott, 2019, p.8).

He also Challenges Gaver (2011) transient nature of design outputs - The idea that Research through design is possibly about the process and the type of object produced. And concludes with;

"Once the designer has created the new object (e.g. an iFloor) they still have to switch hats, so speak, and observe and report as a form of scientist. They make accurate observations, record the data, analyse it and then propose what are hopefully falsifiable claims about the world e.g. a particular way of designing will achieve particular results" (Harriott, 2019, p. 9).

He also struggles with Cross notion of tacit knowledge "Unless tacit knowledge is elevated to the same level as explicit or communicable knowledge, the idea that there is a designerly way of knowing is either an unsupported or a weak claim" (Harriott, 2019, p. 9). Tacit knowledge problematics have also been challenged recently by Michael Meyer and Don Norman (2020).

The fundamental problem with Archer, Cross, and Frayling, is that all of them missed one fundamental variable; time. This is surprising, as this variable was introduced by John Chris Jones in 1970.

\subsection{John Christ Jones}

John Chris Jones, in his seminal book Design methods, postulated that design was different from the arts, sciences, and mathematics. In response to the question "Is designing an art, a science or a form of mathematics?" Jones responded:

"The main point of difference is that of timing. Both artists and scientists operate on the physical world as it exists in the present (whether it is real or symbolic), while mathematicians operate on abstract relationships that are independent of historical time. Designers, on the other hand, are forever bound to treat as real that which exists only in an imagined future and have to specify ways in which the foreseen thing can be made to exist." (Jones, 1992. p. 10)

\subsection{Fernando Galdon \& Ashley Hall}

From this perspective, the authors (2019a) positioned design as a prospective activity in the context of abductive reasoning ((making decisions without having all the information) (Douven, 2011)). In this area, research by Dorst (2010), and more recently Cramer-Petersen et al. (Cramer-Petersen et al., 2018), have concluded that design combines deductive and abductive reasoning; however, in both cases abductive reasoning plays a fundamental role as an initiator of the design activity.

This intrinsic prospective approach of design, based on abductive reasoning, planning, solution-based problem solving, problem shaping, synthesis, preparedness, readiness and appropriateness in the built environment, determines a different model of knowing. In this scenario, the designer is dealing with wicked problems by accessing areas yet-to-be or not-fully-formed (Rittel \& Webber, 1973; 


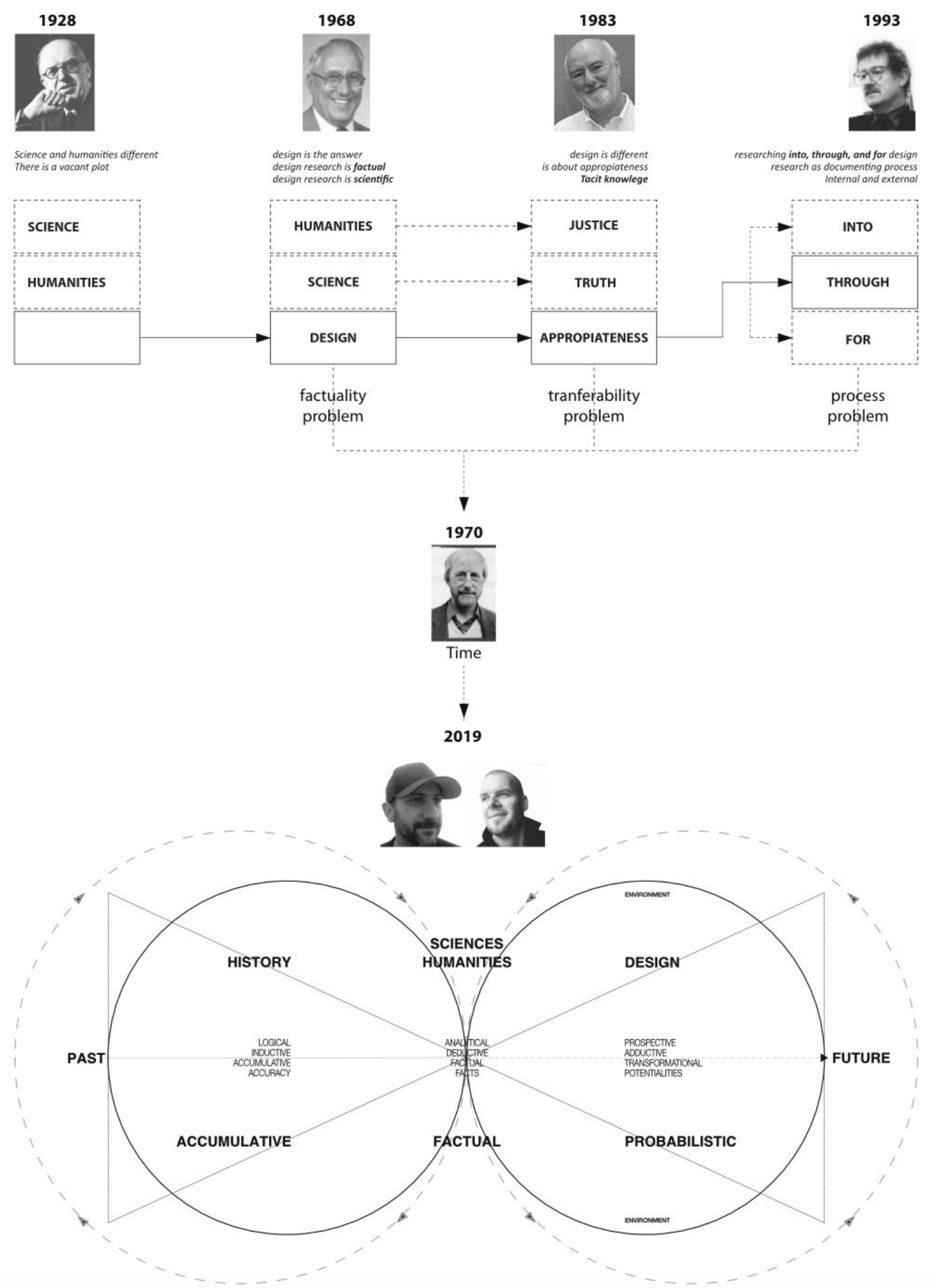

Figure 5. Repositioning design research in design education for the 21 Cth. (Galdon, 2019b). 
Buchanan, 1992; Conklin, 2006). Consequently, its output is based on potentialities, not certainties. We trade some degree of accuracy for access to areas that are yet-to-be or not-fully-formed. Therefore, our output is probabilistic, and research is always preliminary in its nature. Moreover, in exchange we provide guiding knowledge - as Glanville proposed, 'knowledge for' future action and possibilities rather than 'knowledge of' past actions and events (Glanville, 2005). Design research is directional and transformational at its core. In this context, we are more concerned with how things "ought to be'"(Simon, 1995, pp.111-167) instead of how things actually are.

In the prospective framework (Fig. 5) we have proposed that design research can access the future. However, current models of research are limited by the present, both by observation and/or measurement. In order to address this fundamental aspect, we introduced the concept of probabilistic knowledge by building on new approaches in design and economics. Probabilistic knowledge in the context of design research could be defined as the potential impact of transformational initiatives. (See Galdon \& Hall, 2019a for an extended explanation).

In this context, Probabilistic knowledge emerges as an ontological reality to address the intrinsically prospective and abductive nature of design research. Ultimately this approach implies a different form of knowing and aims to position design research as the field best prepared for addressing the future.

\subsection{Design research in design education for the 21Cth}

In 'Towards relational design' (2008), Andrew Blauvelt proposed that we are moving towards a type of design that is relationally based and contextually specific. In his account, he structures the evolution of design into three main epochs: modern design, post-modern design and relational design. Modern design ranges from 1900 to 1950, and focused on forms, which were disseminated rationally and potentially universally. Post-modern design ranged from 1960 to mid 90s, and focused on design's meaning-making potential, symbolic value, semantic dimension and narrative potential. Finally, relational design ranges from the mid 90s to the present, and focuses on effects on users, pragmatic and programmatic constraints, rhetorical impact, and the ability to facilitate social interactions. He presents IDEO and Anthony Dunne and Fiona Raby as primary practitioners in this new evolution. In his account, he describes relational design as including performative, pragmatic, programmatic, process-oriented, open-ended, experiential and participatory elements, moving away from designing discrete objects "to the creation of systems and more open-ended frameworks for engagement: designs for making designs" (Blauvelt, 2008).

If the first wave of design offered us a multiplicity of forms, and the second a multiplicity of meanings and interpretations, the third wave presented a multiplicity of contingent, boundaried or conditional solutions: open-ended rather than closed systems; real-world constraints and contexts over idealised utopias, or pure forms; relational connections instead of reflexive imbrication; "the end of discrete objects, hermetic meanings, and the beginning of connected ecologies" (Blauvelt, 2008, p.6). In this context, the nature, intentionality and implications of emerging interactions demands a different kind of design and time intervention. In this scenario, design research must address complex systems via prospectivity and emancipation, while dealing with uncertainty, not-fully-knowing, reparation, accountability, and the ubiquity of fluid cyber-blended and hyper-connected ecologies. In this context, this paper advances knowledge by making a fundamental contribution to contextualising Glanville's 'knowledge for' future action and possibilities rather than 'knowledge of' past actions and events (Glanville, 2005). 


\section{Conclusions}

Archer's doctoral work attempted to explain design as a special branch of science (but, usefully failed to do so) (Boyd Davis, 2016). Nigel Cross evolution framed the three cultures in terms of output, and presented an evolution to Archer in terms of the knowledge generated, which he framed as tacit knowledge. This reframing aimed to address what we characterise as the problem of factuality.

Tacit knowledge was introduced by Michael Polanyi (1958) as a type of knowledge that is difficult to transfer to another person by means of writing or verbalising. This model of Tacit knowledge was then used by Christopher Frayling to frame an evolution to Nigel Cross model by introducing three new possibilities; Research into Design, Research through design, and Research for design. Frayling model offered a way-out to the tacit conundrum in the form of Research through design combining internal and external perspectives. This category would allow us to integrate design research into stablished models of research while maintaining its integrity which he positioned around the process. This reframing aimed to address the implications of tacit knowledge in what we characterise as the problem of transferability.

Research through design, as described by Frayling, focuses on material science (therefore making a contribution to science, not design), or development work and documentation (therefore, operating as a type of scientific model or making a contribution to the means of archiving). All these elements create a final problem; the problem of process, where design becomes a process/tool/method to develop and document research. If design is a tool/process/method, then, is not a field or culture. This approach is a de facto dissolution of design as a constitutive culture. And, if this process is fundamentally scientific, then, there is not distinctiveness in design research.

Furthermore, any acknowledgement of research as a contribution to knowledge in the field is missing from Frayling account. What constitute the act of academic research is a double-side process of searching and finding. You need to search, but you need to find, and what you find needs to be novel and make a contribution to stablished knowledge.

The fundamental problem with Archer, Cross, and Frayling propositions is that all of them missed one fundamental variable; time. This is surprising, as this variable was introduced by John Chris Jones in 1970. This aspect forced them to operate in the present, and fundamentally, prevented them to understand design as a future-led activity operating around notions of prospectivity, abductivity, contextuality, and probabilism. These categories enable Archer's third culture to be fully operational in the context of research. This model does not aim to prevent researchers to implement research into or through design, it just clarifies where the contribution is made.

The model presented by the authors (2019a) aligns with Cross aforementioned functional claim (appropriateness), and consolidates Archer's design distinctive position, different from the sciences, and humanities. It positions the ontological nature of design knowledge as probabilistic. This reposition emancipates design research from the present, thus overcoming the scientific/tacit paradigms, and liberates design research to operate independently in its future-led, prospective, and transformational nature focused on world-making. But this independence, which is capital to constitute its culture distinctiveness, does not mean insolation, quite the contrary. It facilitates interdependences and collaborations by clarifying what we do to others. Now we can sit in front of a scientist, sociologist or anthropologist and explain what is what we do, exactly as when a sociologist and a scientist sit in front of each other and explain to each other the scientific method, or field work. We are prospective thinkers using abductive reasoning to generate potentialities to transform society and the build environment by prototyping objects/services/actions (World-making). 
In the past we were enclosed in workshops and studios, and now we integrate others in the process, but our expertise, distinctiveness, and culture is to lead this prospective process where practice shape fragmented territories, in dialogue, in solidarity, collectively constituted, and transformational. This is what we do that nobody else can. Design research is directional and transformational at its core, and we are consummated experts in this process.

\section{References}

Archer, L. B. (1978) Time for a revolution in art and design education. RCA Papers No. 6. London: Royal College of Art, London.

Boyd Davis, S., Gristwood, S. (2016). The structure of design processes: ideal and reality in Bruce Archer's 1968 doctoral thesis. In: Proceedings of DRS 2016, Design

Buchanan, R., (1992) Wicked problems in design thinking, Design Issues, 8(2) (Spring), 5-21.

Conklin, J., (2006) Dialogue mapping: building shared understanding of wicked problems. Chichester: John Wiley \& Sons.

Cramer-Petersen, C. L., Christensen, B. T., \& Ahmed-Kristensen, S. (2019). Empirically analysing design reasoning patterns: abductive-deductive reasoning patterns dominate design idea generation. Design Studies, 60, 39-70. DOI: 10.1016/j.destud.2018.10.001

Cross, $\mathrm{N}$ (2001) Designerly ways of knowing: design discipline versus design science. Design Issues Vol 17, No. 3. pp 49-55. DOI: 10.1162/074793601750357196

Douven, I. (2011). Abduction. The Stanford encyclopedia of philosophy (Spring 2011 Edition), Edward N. Zalta ed. Accessed 21/04/2018. Available on: plato.stanford.edu/archives/spr2011/entries/abduction

Dorst, K. (2010). The nature of design thinking. In: DTRS8 Interpreting Design Thinking: Design Thinking Research Symposium Proceedings, 2010, pp. 131 - 139

Frayling, C. (1993). Research in art and design, RCA Research Papers,1, 1

Galdon, F., Hall, A. (2019a). The ontological nature of design; prospecting new futures through probabilistic knowledge. In: Design Research for Change Symposium. Design Museum, London

Galdon, F., Hall, A. \& Wang, S. J. (2019b). Prospective design: A future-led mixed-methodology to mitigate unintended consequences. In: Proceedings of the International Association of Societies of Design Research Conference IASDR2019, The University of Manchester, UK.

Gaver, W. (2012) What should we expect from research through design? CHI '12 Proceedings of the SIGCHI Conference on Human Factors in Computing Systems Pages 937-946

Glanville, R. (2005). Design propositions. In: M. Belderbos and J. Verbeke, eds. The unthinkable doctorate: Brussels: Sint Lucas.

Guerra, L. (2021). Call for Papers: Inmaterial vol. 6, no. 11: Design Research. Practices and Forms. https://arthist.net/archive/33470

Herriott, R. (2019). what kind of research is research through design? In: Proceedings of the International Association of Societies of Design Research Conference IASDR2019, The University of Manchester, UK.

Jones, J. C. (1992). Design methods. New York: Van Nostrand Reinhold.

Meyer, M., and Norman, D. (2020). Changing Design Education for the 21st Century. The Journal of Design, Economics, and Innovation. Volume 6, Issue 1, Spring 2020, Pages 13-49. https://doi.org/10.1016/j.sheji.2019.12.002

Polanyi, M. (1958). Personal Knowledge: Towards a Post-Critical Philosophy. Chicago: University of Chicago Press. ISBN 0-226-67288-3.

Polanyi, M. (1966). The Tacit Dimension. Chicago: University of Chicago Press. p. 4. 
Rittel, H.W.J. \& Webber, (1973). Dilemmas in a General Theory of Planning, M.M. Policy Sci 4: 155.

Simon, H. (1996). The sciences of the artificial. Cambridge, MA: MIT Press.

Snow, C. P. (1959). The two cultures and scientific revolution. New York: Cambridge University Press, 2013.

Author Bios:

Fernando Galdon: Fernando is a Ph.D. candidate concluding a doctoral programme in Global Innovation Design at the Royal college of Art. He is also a Lecturer in design innovation at the Royal College of Art and Imperial college double master in Innovation Design Engineering. Fernando's research focuses on design theory, trust, and applied ethics.

Ashley Hall: Ashley is Professor of Design Innovation at the Royal College of Art where he leads postgraduate research for the design school and the MRes in Healthcare Design. Ashley researches innovation methods, experimental design, design for safety, design pedagogy, globalisation design and cultural transfer. 